Ferrata Storti Foundation

\title{
Impact of treatment with iron chelation therapy in patients with lower-risk myelodysplastic syndromes participating in the European MDS registry
}

Haematologica 2020

Volume 105(3):640-651

\section{Correspondence:}

THEO DE WITTE

t.dewitte@ncmls.ru.nl

Received: November 19, 2018.

Accepted: July 4, 2019.

Pre-published: July 5, 2019.

doi:10.3324/haematol.2018.212332

Check the online version for the most updated information on this article, online supplements, and information on authorship \& disclosures: www.haematologica.org/content/105/3/640

\section{(C)2020 Ferrata Storti Foundation}

Material published in Haematologica is covered by copyright. All rights are reserved to the Ferrata Storti Foundation. Use of published material is allowed under the following terms and conditions:

https://creativecommons.org/licenses/by-nc/4.0/legalcode. Copies of published material are allowed for personal or internal use. Sharing published material for non-commercial purposes is subject to the following conditions:

https://creativecommons.org/licenses/by-nc/4.0/legalcode, sect. 3. Reproducing and sharing published material for commercial purposes is not allowed without permission in writing from the publisher.
Marlijn Hoeks, ${ }^{1,2} \mathrm{Ge} \mathrm{Yu},{ }^{3}$ Saskia Langemeijer, ${ }^{4}$ Simon Crouch, ${ }^{3}$ Louise de Swart, ${ }^{4}$ Pierre Fenaux, ${ }^{5}$ Argiris Symeonidis, ${ }^{6}$ Jaroslav Čermák, Eva Hellström-Lindberg, ${ }^{8}$ Guillermo Sanz, ${ }^{9}$ Reinhard Stauder, ${ }^{10}$ Mette Skov Holm,${ }^{11}$ Moshe Mittelman, ${ }^{12}$ Krzysztof Mądry, ${ }^{13}$ Luca Malcovati, ${ }^{14}$ Aurelia Tatic, ${ }^{15}$ Antonio Medina Almeida, ${ }^{16}$ Ulrich Germing, ${ }^{17}$ Aleksandar Savic, ${ }^{18}$ Njetočka Gredelj Šimec, ${ }^{19}$ Dominic Culligan, ${ }^{20}$ Raphael Itzykson, ${ }^{5}$ Agnes Guerci-Bresler,${ }^{21}$ Borhane Slama, ${ }^{22}$ Arjan van de Loosdrecht, ${ }^{23}$ Corine van Marrewijk ${ }^{4}$ Jackie Droste,${ }^{4}$ Nicole Blijlevens, ${ }^{4}$ Marian van Kraaij, ${ }^{24}$ David Bowen, ${ }^{25}$ Theo de Witte ${ }^{26}$ and Alex Smith ${ }^{3}$ on behalf of the EUMDS Registry Participants

${ }^{1}$ Centre for Clinical Transfusion Research, Sanquin Research, Leiden, the Netherlands; ${ }^{2}$ Department of Clinical Epidemiology, Leiden University Medical Center, Leiden, the Netherlands; ${ }^{3}$ Epidemiology and Cancer Statistics Group, Department of Health Sciences, University of York, York, USA; ' ${ }^{4}$ epartment of Hematology, Radboud University Medical Center, Nijmegen, the Netherlands; ${ }^{5}$ Service d'Hématologie, Hôpital Saint-Louis, Assistance Publique des Hôpitaux de Paris and Université Paris 7, Paris, France; ${ }^{6}$ Department of Medicine, Division of Hematology, University of Patras Medical School, Patras, Greece; ${ }^{7}$ Department of Clinical Hematology, Institute of Hematology and Blood Transfusion, Praha, Czech Republic; ${ }^{8}$ Department of Medicine, Division of Hematology, Karolinska Institutet, Stockholm, Sweden; ${ }^{9}$ Department of Haematology, Hospital Universitario y Politécnico La Fe, Valencia, Spain; ${ }^{10}$ Department of Internal Medicine V (Haematology and Oncology), Innsbruck Medical University, Innsbruck, Austria; ${ }^{11}$ Department of Haematology, Aarhus University Hospital, Aarhus, Denmark; ${ }^{12}$ Department of Medicine A, Tel Aviv Sourasky (Ichilov) Medical Center and Sackler Medical Faculty, Tel Aviv University, Tel Aviv, Israel; ${ }^{13}$ Department of Haematology, Oncology and Internal Medicine, Warszawa Medical University, Warszawa, Poland; ${ }^{14}$ Department of Hematology Oncology, Fondazione Istituto Di Ricovero e Cura a Carettere Scientifico, Policlinico San Matteo, University of Pavia, Pavia, Italy; ${ }^{15}$ Center of Hematology and Bone Marrow Transplantation, Fundeni Clinical Institute, Bucharest, Romania; ${ }^{16}$ Department of Hematology, Hospital da Luz, Lisbon, Portugal; ${ }^{17}$ Department of Haematology, Oncology and Clinical Immunology, Universitätsklinik Düsseldorf, Düsseldorf, Germany; ${ }^{18} \mathrm{Clinic}$ of Hematology - Clinical Center of Vojvodina, Faculty of Medicine, University of Novi Sad, Novi Sad, Serbia; ${ }^{19}$ Department of Internal Medicine, Division of Hematology, Merkur University Hospital, Zagreb, Croatia; ${ }^{20}$ Department of Haematology, Aberdeen Royal Infirmary, Aberdeen, UK; ${ }^{21}$ Service d'Hématologie, Centre Hospitalier Universitaire Brabois Vandoeuvre, Nancy, France; ${ }^{22}$ Service d'Hématologie, Centre Hospitalier d'Avignon, Avignon, France; ${ }^{23}$ Department of Hematology - Cancer Center Amsterdam VU University Medical Center, Amsterdam, the Netherlands; ${ }^{24}$ Unit Transfusion Medicine, Sanquin Blood Bank, Amsterdam, the Netherlands; ${ }^{25}$ St. James's Institute of Oncology, Leeds Teaching Hospitals, Leeds, UK and ${ }^{26}$ Department of Tumor Immunology - Nijmegen Center for Molecular Life Sciences, Radboud University Medical Center, Nijmegen, the Netherlands

\section{ABSTRACT}

I ron overload due to red blood cell $(\mathrm{RBC})$ transfusions is associated with morbidity and mortality in lower-risk myelodysplastic syndrome (MDS) patients. Many studies have suggested improved survival after iron chelation therapy (ICT), but valid data are limited. The aim of this study was to assess the effect of ICT on overall survival and hematologic improvement in lower-risk MDS patients in the European MDS registry. We compared chelated patients with a contemporary, non-chelated control group within the European MDS registry, that met the eligibility criteria for starting iron chelation. A Cox proportional hazards model was used to assess overall survival (OS), treating receipt of chelation as a time-varying variable. Additionally, chelated and non-chelated patients were compared 
using a propensity-score matched model. Of 2,200 patients, 224 received iron chelation. The hazard ratio and 95\% confidence interval for OS for chelated patients, adjusted for age, sex, comorbidity, performance status, cumulative RBC transfusions, Revised-International Prognostic Scoring System (IPSS-R), and presence of ringed sideroblasts was 0.50 (0.34-0.74). The propensity-score analysis, matched for age, sex, country, RBC transfusion intensity, ferritin level, comorbidity, performance status, and IPSS-R, and, in addition, corrected for cumulative RBC transfusions and presence of ringed sideroblasts, demonstrated a significantly improved OS for chelated patients with a hazard ratio of $0.42(0.27-0.63)$ compared to non-chelated patients. Up to $39 \%$ of chelated patients reached an erythroid response. In conclusion, our results suggest that iron chelation may improve OS and hematopoiesis in transfused lower-risk MDS patients. This trial was registered at clinicaltrials.gov identifier: 00600860.

\section{Introduction}

Myelodysplastic syndromes (MDS) comprise a heterogeneous group of clonal hematopoietic stem cell disorders characterized by abnormal differentiation and maturation of hematopoietic cells, bone marrow (BM) failure and genetic instability, with an enhanced risk of progressing to acute myeloid leukemia (AML). ${ }^{1}$ Iron overload (IOL), as a consequence of frequently administered red blood cell transfusions (RBCT) and/or ineffective erythropoiesis, is a common finding in MDS. The effects of toxic iron species in other iron loading diseases, such as primary hemochromatosis, thalassemia and sickle cell anemia are well known, but the consequences in MDS are less clear. ${ }^{2-4}$ With an expected median survival of 2.4-11.8 years in lower risk MDS (LR-MDS) patients, ${ }^{5}$ these patients are prone to long-term accumulation of iron due to RBCT as well as direct iron toxicity due to the formation of reactive oxygen species (ROS). ${ }^{6}$

Several studies have reported beneficial effects of iron chelation therapy (ICT) on overall survival (OS) and other clinical outcomes in MDS patients with IOL. ${ }^{7-10}$ However, valid data on the effect of ICT are limited, as most studies are carried out in small or highly selected patient groups, or suffer from serious methodological problems such as confounding by indication. Performing a randomized, controlled trial to explore this is cumbersome due to patients' widespread belief in the beneficial effects of ICT and also the personal opinion of many treating physicians, which may negatively affect enrollment. Likewise, patients included in a randomized, controlled trial do not generally reflect the actual LR-MDS patient group, which is usually made up of elderly patients with multiple comorbidities.

In addition to the possible beneficial effects of ICT on $\mathrm{OS}$, there is increasing evidence to indicate hematologic improvement in patients during treatment with iron chelators. ${ }^{11-16}$ Alongside improvement in hemoglobin, platelet, and neutrophil levels, transfusion independence is achieved in a minority of chelated patients. ${ }^{11,12,14}$ The underlying mechanisms are still unclear. ${ }^{17}$

The aim of this study was to evaluate the effect of ICT on OS, hematologic improvement, and ferritin levels in lower risk MDS patients in the European MDS (EUMDS) Registry.

\section{Methods}

The EUMDS registry prospectively collects observational data on LR-MDS patients from 142 centers in 16 countries in Europe and Israel. Patients were included within 100 days of MDS diagnosis according to the World Health Organization 2001 classification, restricted to patients with a low or intermediate-1 score according to the International Prognostic Scoring System (IPSS).${ }^{18}$ IPSS was the current prognostic indicator at the start of the registry, in accordance with the currently used prognostic score; the Revised-IPSS (IPSS-R) was reconstructed afterwards The ethics committees of all participating centers approved the protocol and all patients provided written informed consent. Data were collected at baseline and at each 6-monthly routine outpatient follow-up visit. Data were collected on: comorbidity, transfusion history, use of iron chelators (agent, time frame; no drug doses or schedules were collected), peripheral blood values, conventional iron parameters (e.g. serum ferritin), bone marrow pathology, and progression to higher-risk MDS or AML. Subjects were prospectively followed until death, loss to follow up, or withdrawal of informed consent.

In Europe, three iron chelators are available for treatment of secondary IOL, but availability varies between countries. We analyzed all patients, chelated or non-chelated, who are eligible for receiving ICT based on at least one criterion for starting ICT (cumulative $\geq 15 \mathrm{RBC}$ units, RBCT intensity of $\geq 1$ unit/month during a 6-month period, or serum ferritin level $>1000 \mu \mathrm{g} / \mathrm{L}$ ), thereby preventing immortal time bias. As chelated and nonchelated patients may differ in characteristics that affect outcome, two different approaches were performed in order to control for potential bias: 1) analysis of all eligible chelated and nonchelated patients using receipt of ICT as a time-varying co-variate, adjusting for co-variates related to both receiving ICT and OS: sex, age, comorbidity, performance status, RBCT intensity, number of units transfused, IPSS-R, and presence of ringed sideroblasts; 2) Propensity Score (PS), i.e. conditional probability for being treated with ICT on the basis of patient characteristics, matching of the same group. Variables included in the PS were: age, sex, country, RBCT intensity, ferritin level, MDS comorbidity index, performance status, and IPSS-R. A 3-to-1 nearest neighbor matching method with replacement and caliper (0.2) was applied. ${ }^{19}$ In addition, we used a robust sandwich estimator to correct for intra-individual correlation of multiply used controls. Further details on the PS matching are provided in the Online Supplementary Methods. ${ }^{20-22}$ OS was defined as the time from eligibility for ICT to death; subjects still alive were censored at the last follow-up date. Cox proportional hazards regression models and Kaplan-Meier survival curves were applied and hazard ratios (HR) with 95\% confidence intervals ( $95 \% \mathrm{CI})$ were reported. ${ }^{23}$

Erythroid responses were defined as a reduction in RBCT density (number of RBCT over time; see Online Supplementary Methods for definition and details) or as transfusion independency at least once as the transfusion density was reduced to zero, platelet responses were assessed according to the modified 
International Working Group (IWG) criteria. ${ }^{24}$ Ferritin responses were defined as a decrease of $\geq 1000 \mu \mathrm{g} / \mathrm{L}$ or a drop of the serum ferritin value below $1000 \mu \mathrm{g} / \mathrm{L}$.

All analyses were undertaken in Stata 15 (StataCorp, College Station, TX, USA).

\section{Results}

\section{Patient population}

Data were extracted from the EUMDS registry on July $5^{\text {th }} 2017,2,200$ patients, diagnosed between December $3^{\text {rd }}$

Table 1. Baseline characteristics of non-chelated and chelated patients at the check-up prior to reaching the eligibility criteria and estimates of overall survival.

\begin{tabular}{|c|c|c|c|c|c|}
\hline & Non-chelated & Chelated & Deferasirox & Deferoxamine & Deferiprone \\
\hline Total & 490 & 199 & 150 & 36 & 13 \\
\hline N. of countries with chelated patients & $17 / 17$ & $17 / 17$ & $14 / 17$ & $9 / 17$ & $6 / 17$ \\
\hline Mean age at eligible (SD) & $76(10)$ & $70(9)$ & $70(9)$ & $72(9)$ & $70(10)$ \\
\hline \multicolumn{6}{|l|}{ Time from diagnosis (months) } \\
\hline Inclusion, median (p10-p90) & $7(0-35)$ & $8(0-32)$ & $9(0-36)$ & $6(0-30)$ & $7(0-33)$ \\
\hline Inclusion, mean (SD) & $14(16)$ & $13(15)$ & $14(16)$ & $9(11)$ & $12(13)$ \\
\hline Chelation median (p10-p90) & NA & $17(4-46)$ & $17(4-47)$ & $13(2-39)$ & $22(5-51)$ \\
\hline Chelation mean (SD) & NA & $21(17)$ & $21(18)$ & $17(13)$ & $26(18)$ \\
\hline
\end{tabular}

Number of units transfused

Median (range)

Median at start of chelation (range)

$4.0(1.0-33.0)$

$2.0(0.0-28.0)$

$2.5(0.0-28.0)$

$3.0(0.0-18.0)$

$2.0(0.0-8.0)$

Ferritin $(\mu \mathrm{g} / \mathrm{L})$

Median (p10-p90)

$\mathrm{NA}$

$13.0(2.0-91.0)$

$12.0(2.0-75.0)$

$10.5(2.0-75.0)$

$24.5(2.0-91.0)$

Media

Median at start of chelation (p10-p90)

547.0

675.0

(116.0 - 1384.0)

(256.0 - 1573.0)

NA

1221.0

682.0

525.0

$(475.8-3000.0)$

(264.0 - 1600.0)

(256.0 - 1920.0)

(190.5-918.1)

Comorbidity (MDSCI)

Low risk

Intermediate risk

High risk

$308 \quad 63.2 \%$

$150 \quad 75.8 \%$

$(449.3-2832.0)$

1173.0

2202.0

$(335.0-3000.0)$

$(475.8-4900.0)$

Performance status

Unable to care for self

$14930.6 \%$

$43 \quad 21.7 \%$

$118 \quad 79.2 \%$

$23 \quad 63.9 \%$

$969.2 \%$

$30 \quad 6.2 \%$

$5 \quad 2.5 \%$

$28 \quad 18.8 \%$

$1130.6 \%$

4 30.8\%

$8+2.0 \%$

$32.0 \%$

$25.6 \%$

$0 \quad 0.0 \%$

Unable to work

$\begin{array}{rr}8 & 2.0 \% \\ 132 & 32.3 \%\end{array}$

$10.6 \%$

$10.8 \%$

$\begin{array}{ll}0 & 0.0 \%\end{array}$

$\begin{array}{ll}0 & 0.0 \%\end{array}$

Able to work and normal activity

$26965.8 \%$

$36 \quad 20.2 \%$

$21 \quad 15.9 \%$

$1234.3 \%$

$3 \quad 27.3 \%$

Prognostic indicator (IPSS-R)

Reaching criteria (LOCF***)

Very low

Low

Intermediate

High

$14179.2 \%$

$110 \quad 83.3 \%$

$23 \quad 65.7 \%$

$8 \quad 72.7 \%$

Very high

Duration of treatment with chelation (months)

Median (p10-p90)

Ever received ESA

No

Yes

$48 \quad 13.4 \%$

$22 \quad 13.5 \%$

$18 \quad 14.6 \%$

$26.9 \%$

$2 \quad 18.2 \%$

$19955.6 \%$

$95 \quad 58.3 \%$

$66 \quad 53.7 \%$

$2172.4 \%$

$872.7 \%$

$11131.0 \%$

$46 \quad 28.2 \%$

$39 \quad 31.7 \%$

$620.7 \%$

$19.1 \%$

$38 \quad 10.6 \%$

$95.5 \%$

$6 \quad 4.9 \%$

$3 \quad 10.3 \%$

$\begin{array}{lll}0 & 0.0 \%\end{array}$

$3 \quad 0.8 \%$

$10.6 \%$

$10.8 \%$

$0.0 \%$
0.00

$0 \quad 0.0 \%$

NA $\quad 13(3-41)$

$14(3-41)$

$9(1-34)$

$13(2-30)$

$312 \quad 63.7 \%$

$115 \quad 57.8 \%$

$85 \quad 56.7 \%$

$22 \quad 61.1 \%$

$8 \quad 61.5 \%$

Ever received hypomethylating

No
Yes

$178 \quad 36.3 \%$

$84 \quad 42.2 \%$

$65 \quad 43.3 \%$

$14 \quad 38.9 \%$

$538.5 \%$

$460 \quad 93.9 \%$

$184 \quad 92.5 \%$

$136 \quad 90.7 \%$

$36 \quad 100.0 \%$

$1292.3 \%$

Ever received lenalidomide

$30 \quad 6.1 \%$

$15 \quad 7.5 \%$

$14 \quad 9.3 \%$

$\begin{array}{ll}0 & 0.0 \%\end{array}$

\begin{tabular}{|c|c|c|c|c|c|}
\hline No & $467 \quad 95.3 \%$ & $17989.9 \%$ & $13690.7 \%$ & $3391.7 \%$ & $1076.9 \%$ \\
\hline Yes & $234.7 \%$ & $20 \quad 10.1 \%$ & $149.3 \%$ & $38.3 \%$ & $323.1 \%$ \\
\hline
\end{tabular}

Overall Survival (OS)*

Unadjusted

Adjusted**

$1 \quad 0.57 \quad(0.45-0.73)$

1

$1.99(1.18-3.35) \quad 0.42(0.10-1.71)$

*Hazard Ratios (HR) and 95\% Confidence Intervals (CI) were estimated using receipt of chelation as a time-varying co-variate. ** Adjusted by age at eligibility,sex, comorbidity Performance Status, number of units transfused, Revised-International Prognostic Scoring System (IPSS-R), and ringed sideroblasts present. ***LOCF: last observation carried forward (only for cytogenetics and bone marrow blasts); SD: Standard Deviation; RBCT: red blood cell transfusion; MDSCI: Myelodysplastic Syndrome Specific Comorbidity Index; ESA: erythropoiesis stimulating agents; NA: not applicable. 
2007 and April 25 2017 , had been registered, of which 1,161 patients received at least one RBCT and 224 patients received iron chelation therapy (ICT) (Figure 1). A small proportion of patients had received ICT without being transfused or prior to starting RBCT, these subjects generally had a high ferritin level and were excluded from subsequent analyses. Of the 1,161 transfused patients, 850 patients had been transfused for a duration of $\geq 2$ months. Out of these 850 patients, 689 met the eligibility criteria. Online Supplementary Figure $S 1$ summarizes the number of patients who reached each criterion. At the time of analysis, 236 patients were deceased (154 nonchelated, 82 chelated) and nine patients progressed to high-risk MDS or AML (4 non-chelated, 5 chelated).

\section{Comparing outcome of chelated versus non-chelated patients using iron chelation therapy as a time-dependent variable}

Table 1 shows the characteristics of the 689 patients at the check-up prior to meeting one of the eligibility criteria; the date of this check-up is when the patients entered this analysis. Mean age of the 199 chelated patients was 70 years and these patients were younger than the nonchelated patients (mean age was 76 years). Median time from date of diagnosis to date of meeting the eligibility criteria was seven months in the non-chelated and eight months in the chelated subjects. The median follow-up period from study entry for chelated and non-chelated patients was 39.4 months (range 4.1-106.6 months) and 27.1 months (range 2.5-105.6 months), respectively. Nonchelated subjects had a higher number of cumulative units transfused than chelated subjects ( 4 vs. 2 units) at time of inclusion and, on average, chelated patients had 13 units transfused prior to commencing ICT. The latter had a higher median ferritin level recorded at baseline $(675 \mu \mathrm{g} / \mathrm{L}$ vs. $547 \mu \mathrm{g} / \mathrm{L})$, and this had increased to $1,221 \mu \mathrm{g} / \mathrm{L}$ prior to start of ICT. While non-chelated and chelated subjects had similar IPPS-R scores, chelated patients had fewer co-morbidities as measured by the MDS-CI score and a better performance status as measured by Karnofsky
Performance Status. OS was estimated using receiving ICT as a time-dependent variable, hence the number of patients reported in the risk table in Figure 2 reflects the time when a subject commences ICT. The hazard ratio for OS in the univariate analysis was 0.57 (95\% CI: 0.45-0.73) (Table 1 and Figure 2). This benefit increased when adjusted for the factors in Table 1 and the following variables: sex, RBCT intensity, and the presence of ringed sideroblasts (HR: 0.50, 95\% CI: 0.34-0.74). No statistically significant interactions were detected when using a sophisticated prediction-type model. When we restricted the analysis to patients who were treated with deferasirox (the largest group), thereby excluding possible differences between patients using different chelators, the crude HR for OS was 0.53 (95\% CI: 0.40-0.69) and the adjusted HR for OS was 0.38 (95\%CI: 0.24-0.60). Out of the 199 chelated patients, 150 received deferasirox as the initial chelator, 36 deferoxamine, and 13 deferiprone, and differences were seen in the baseline characteristics by type of chelator with deferasirox-treated patients being younger and fitter. Twenty-two patients switched from one chelator to another, or were treated with all three chelators consecutively (Online Supplementary Table S1), but usually the treatment period of the second chelator was shorter than the treatment period of the first chelator. The median time on chelation for all 199 patients was 13 months (range 341 months) and patients who were initially treated with deferoxamine had inferior OS compared to deferasiroxtreated patients (adjusted HR: 2.46, 95\% CI: 1.12-5.41) (Table 1). The OS of the deferoxamine-treated patients was similar to non-chelated patients (adjusted HR: 0.98, 95\% CI: 0.52-1.86).

\section{Matching of chelated and non-chelated patients by propensity scores}

The variables used in the propensity score matching are described in Online Supplementary Table S2 for all eligible patients by chelation status, initially excluding any missing variables and then after multiple imputation (MI). Along with the factors already shown in Table 1, there

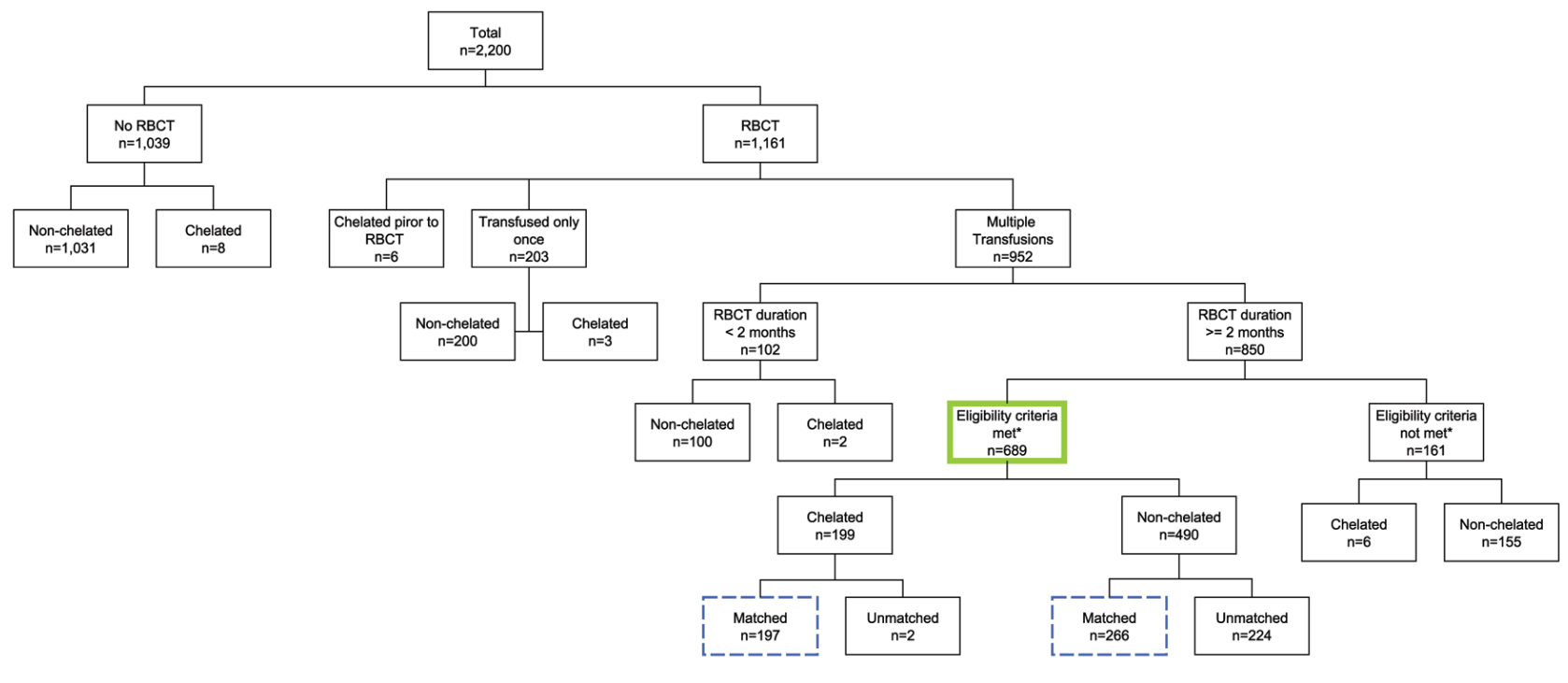

Figure 1. Number of registry patients by transfusion and chelation status. *Cumulative red blood cell transfusion (RBCT) units $>15$ or RBCT intensity of $>1$ RBC unit/month or serum ferritin $>1000 \mu \mathrm{g} / \mathrm{L}$. 
was a difference by country as to whether a patient was treated with ICT; patients in the UK were less likely to be treated.

The overlap of propensity scores of both groups (chelated and non-chelated), which is essential for PS matching, was good for the majority of the patients (Online Supplementary Figure S2). The matched MI dataset included 197 of 199 chelated cases and identified 591 non-chelated controls. There were no differences by sex, RBCT intensity, cumulative RBCT units, serum ferritin levels, comorbidity, performance status, IPSS-R, presence of ringed sideroblasts, quality of life (QoL), and country between both groups (Table 2). Figure 3 shows the unadjusted survival plot by ICT status with receiving ICT as a time-dependent variable for the matched patients. A multivariate Cox proportional hazard model was used to adjust for potential confounders (age, sex, comorbidity, performance status, monthly RBCT intensity, number of $\mathrm{RBC}$ units transfused, IPSS-R, and presence of ringed sideroblasts). The estimated crude and adjusted hazard ratios were 0.70 (95\% CI: 0.51-0.95) and 0.42 (0.27-0.63), respectively (Table 2 ) and the adjusted survival curve is shown in Figure 4. When we again restricted the analysis to the deferasirox-treated patients, the crude HR for OS was 0.63 (95\% CI: $0.45-0.88)$ and the adjusted HR was 0.34 (95\% CI: 0.22-0.53).

The distribution of erythropoiesis-stimulating agent (ESA) and lenalidomide-treated patients among chelated and non-chelated patients at time of eligibility were similar in the unmatched and matched sample. A sensitivity analysis excluding the treatment of ESA and lenalidomide showed similar results.

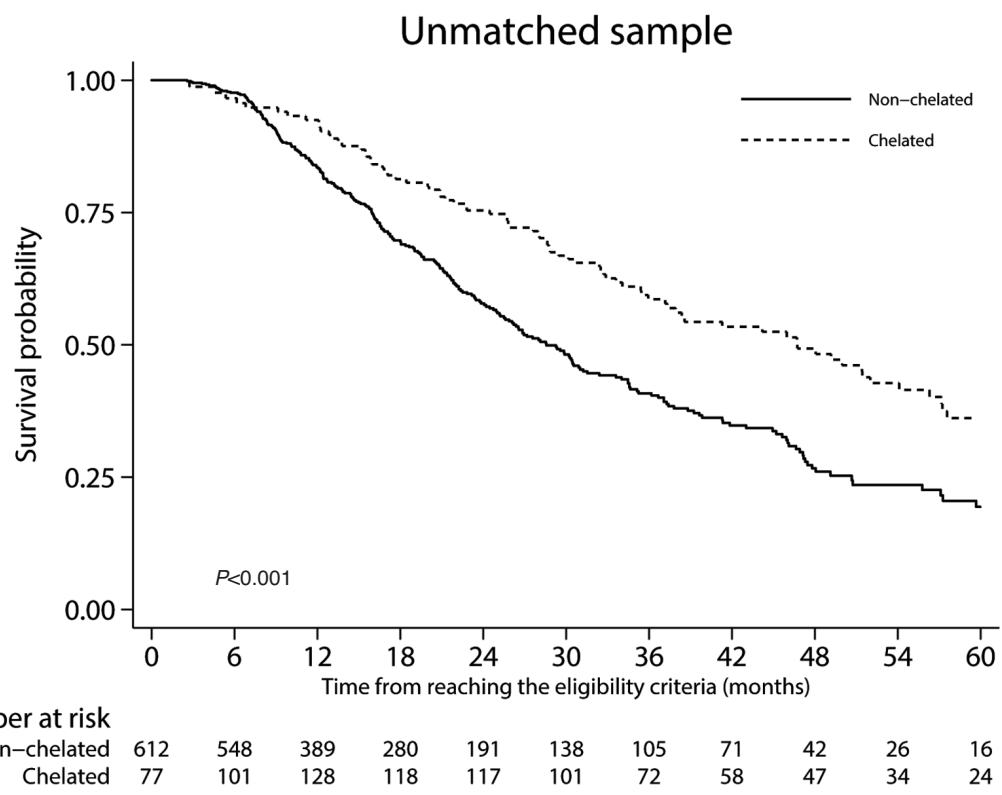

Figure 2. Overall survival by iron chela-

$\begin{array}{rlllllllllll}\text { Number at risk } & & & & & & & \\ \text { Non-chelated } & 612 & 548 & 389 & 280 & 191 & 138 & 105 & 71 & 42 & 26 & 16 \\ \text { Chelated } & 77 & 101 & 128 & 118 & 117 & 101 & 72 & 58 & 47 & 34 & 24\end{array}$

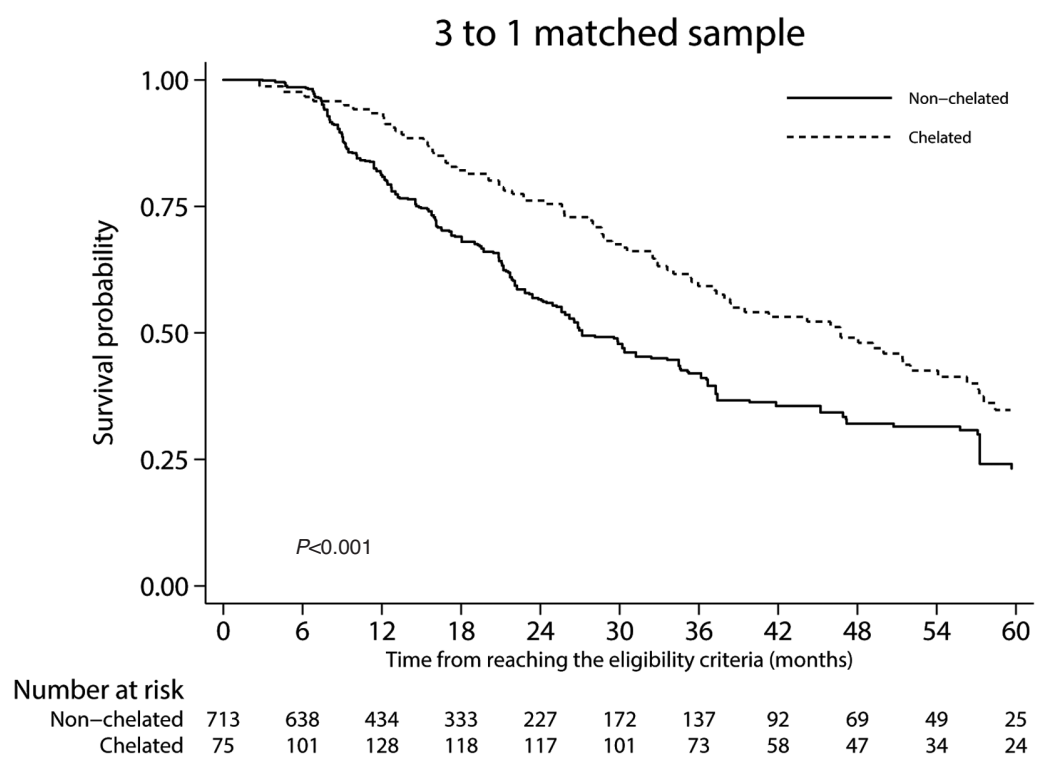

Figure 3. Overall survival by iron chelation therapy as a time-dependent variable in matched patients. 
Table 2. Baseline characteristics for all matched subjects included in the propensity analyses

\begin{tabular}{|c|c|c|c|c|}
\hline \multirow[t]{2}{*}{ Co-variates } & \multirow[b]{2}{*}{$\begin{array}{c}\text { Non-chelated } \\
\mathrm{N}=591\end{array}$} & \multicolumn{3}{|c|}{ Matched* data with imputations** } \\
\hline & & $\begin{array}{l}\text { Chelated } \\
N=197\end{array}$ & \multirow{2}{*}{$\begin{array}{c}P \\
0.364\end{array}$} & \multirow{2}{*}{$\begin{array}{c}\text { Standardized } \\
\text { differences*** } \\
-0.077\end{array}$} \\
\hline Age (years) & 71 (11) & $70 \quad(9)$ & & \\
\hline Sex & & & 0.797 & -0.021 \\
\hline Female & $210 \quad 35.5 \%$ & $72 \quad 36.5 \%$ & & \\
\hline Male & $381 \quad 64.5 \%$ & $125 \quad 63.5 \%$ & & \\
\hline RBCT Intensity (per month) & $0.7(1.0)$ & $0.6(1.0)$ & 0.484 & -0.058 \\
\hline Cumulative RBCT units & $4.5(4.9)$ & $4.3(4.7)$ & 0.570 & -0.047 \\
\hline Ferritin level ( $\mu g / L$, median, p25-p75) & $730.6 \quad(494.6-977.3)$ & $683.6 \quad(504-915.5)$ & 0.328 & -0.086 \\
\hline Comorbidity (MDSCI) & & & 0.965 & -0.004 \\
\hline Low risk & $440 \quad 74.5 \%$ & $15076.1 \%$ & & \\
\hline Intermediate risk & $145 \quad 24.5 \%$ & $42 \quad 21.3 \%$ & & \\
\hline High risk & $61.0 \%$ & $5 \quad 2.5 \%$ & & \\
\hline Performance status & & & 0.279 & 0.090 \\
\hline Unable to care for self & $4 \quad 0.7 \%$ & $10.5 \%$ & & \\
\hline Unable to work & $135 \quad 22.8 \%$ & $38 \quad 19.3 \%$ & & \\
\hline Able to work and normal activity & $45276.5 \%$ & $158 \quad 80.2 \%$ & & \\
\hline Prognostic indicator (IPSS-R) & & & 0.914 & 0.009 \\
\hline Very low & $83 \quad 14.0 \%$ & $22 \quad 11.2 \%$ & & \\
\hline Low & $337 \quad 57.0 \%$ & $120 \quad 60.9 \%$ & & \\
\hline Intermediate & $134 \quad 22.7 \%$ & $45 \quad 22.8 \%$ & & \\
\hline High & $34 \quad 5.8 \%$ & $94.6 \%$ & & \\
\hline Very high & $30.5 \%$ & $10.5 \%$ & & \\
\hline Ring-sideroblast present & & & 0.445 & 0.062 \\
\hline Yes & $41970.9 \%$ & $134 \quad 68.0 \%$ & & \\
\hline No & $17229.1 \%$ & $63 \quad 32.0 \%$ & & \\
\hline Platelet level (10x/L, median, p25-p75) & $162.5 \quad(99.2-294)$ & $224.0(121-324)$ & 0.086 & 0.148 \\
\hline Hemoglobin level (g/dL, median, p25-p75) & $8.8(8.2-9.8)$ & $8.4(7.7-9.5)$ & 0.021 & -0.194 \\
\hline \multicolumn{5}{|l|}{ Quality of Life (EQ-5D) } \\
\hline Index (mean, SD) & $0.7(0.2)$ & $0.7(0.2)$ & 0.186 & 0.125 \\
\hline VAS (mean, SD) & $64.8(21.0)$ & $68.1(19.9)$ & 0.083 & 0.165 \\
\hline Country & & & 0.140 & -0.122 \\
\hline Austria & $25 \quad 4.2 \%$ & $105.1 \%$ & & \\
\hline Croatia & $91.5 \%$ & $10.5 \%$ & & \\
\hline Czech Republic & $58 \quad 9.8 \%$ & $25 \quad 12.7 \%$ & & \\
\hline Denmark & $15 \quad 2.5 \%$ & $84.1 \%$ & & \\
\hline France & $11319.1 \%$ & $40 \quad 20.3 \%$ & & \\
\hline Germany & $23 \quad 3.9 \%$ & $84.1 \%$ & & \\
\hline Greece & $80 \quad 13.5 \%$ & $23 \quad 11.7 \%$ & & \\
\hline Israel & $111.9 \%$ & $5 \quad 2.5 \%$ & & \\
\hline Italy & $111.9 \%$ & $5 \quad 2.5 \%$ & & \\
\hline the Netherlands & $17 \quad 2.9 \%$ & $73.6 \%$ & & \\
\hline Poland & $22 \quad 3.7 \%$ & $8 \quad 4.1 \%$ & & \\
\hline Portugal & $2 \quad 0.3 \%$ & $10.5 \%$ & & \\
\hline Romania & $34 \quad 5.8 \%$ & $115.6 \%$ & & \\
\hline Republic of Serbia & $8 \quad 1.4 \%$ & $21.0 \%$ & & \\
\hline Spain & $11 \quad 1.9 \%$ & $52.5 \%$ & & \\
\hline Sweden & $97 \quad 16.4 \%$ & $20 \quad 10.2 \%$ & & \\
\hline UK & $55 \quad 9.3 \%$ & $189.1 \%$ & & \\
\hline \multicolumn{5}{|l|}{ Overall Survival (OS) } \\
\hline Unadjusted & 1.0 & $0.70(0.51-0.95)$ & & \\
\hline Adjusted**** & 1.0 & $0.42(0.27-0.63)$ & & \\
\hline
\end{tabular}


Impact of iron chelation therapy on hematopoiesis and ferritin levels

Figure 5 shows the changes in transfusion density over eight visits in chelated and non-chelated patients. Fortyeight $(62.3 \%)$ of the 77 responding patients were treated with ESA and 16 (20.8\%) were treated with lenalidomide during chelation therapy. Compared to first check-up, 61 of the 197 chelated patients $(31.0 \%)$ had a reduction in transfusion density, i.e. an absolute decrease, during at least one interval between check-ups, two patients $(1.0 \%)$ maintained the same density throughout, and 134 $(68.0 \%)$ never had a reduction in transfusion density. For those patients who showed a reduction, the average value in the monthly rate was -1.63 units per month (SD: 2.12, median: -0.96) compared to first check-up. Figure
6A shows the monthly RBC transfusion density for chelated patients with and without an erythroid response and non-chelated patients. In terms of becoming transfusion independent, 35 (17.8\%) of the 197 treated patients had at least one interval between check-ups of approximately six months during which they had not received any further transfusions and 19 (9.6\%) of the 197 patients were transfusion independent during more than one interval between check-ups after starting chelation therapy. In total, 54 patients $(27.4 \%$ ) became (temporarily) transfusion independent. In total, 77 chelated patients had an erythroid response: 61 patients had a reduction in transfusion density, and 16 patients who did not have a reduction in transfusion density became transfusion independent during at least one interval between check-ups.

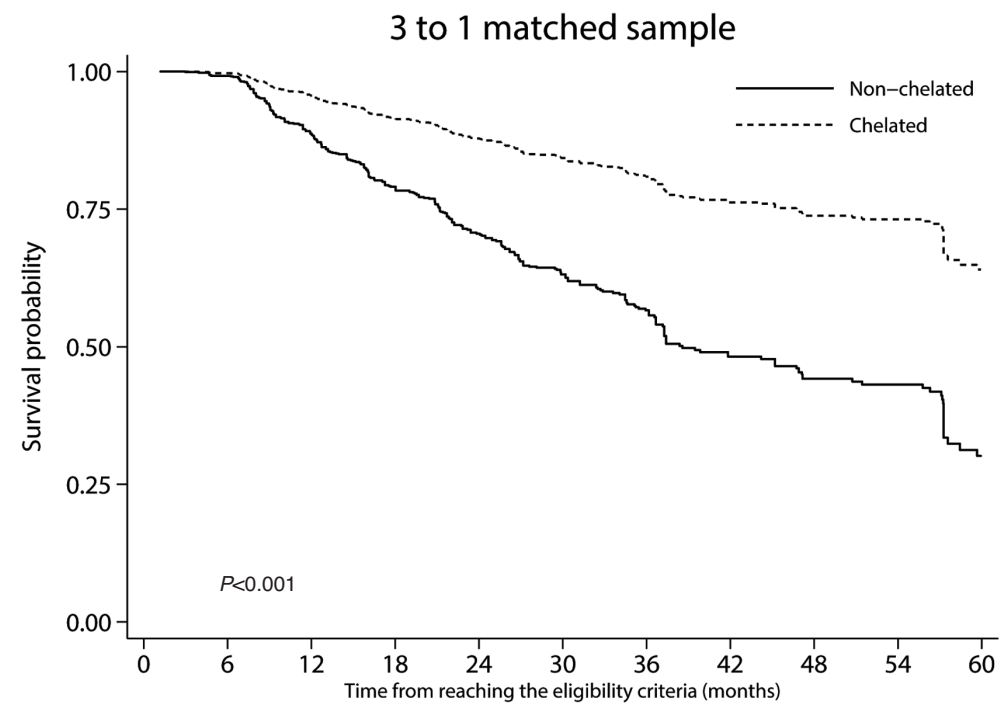

Figure 4. Adjusted overall survival by iron chelation therapy as a time-dependent variable in matched patients.
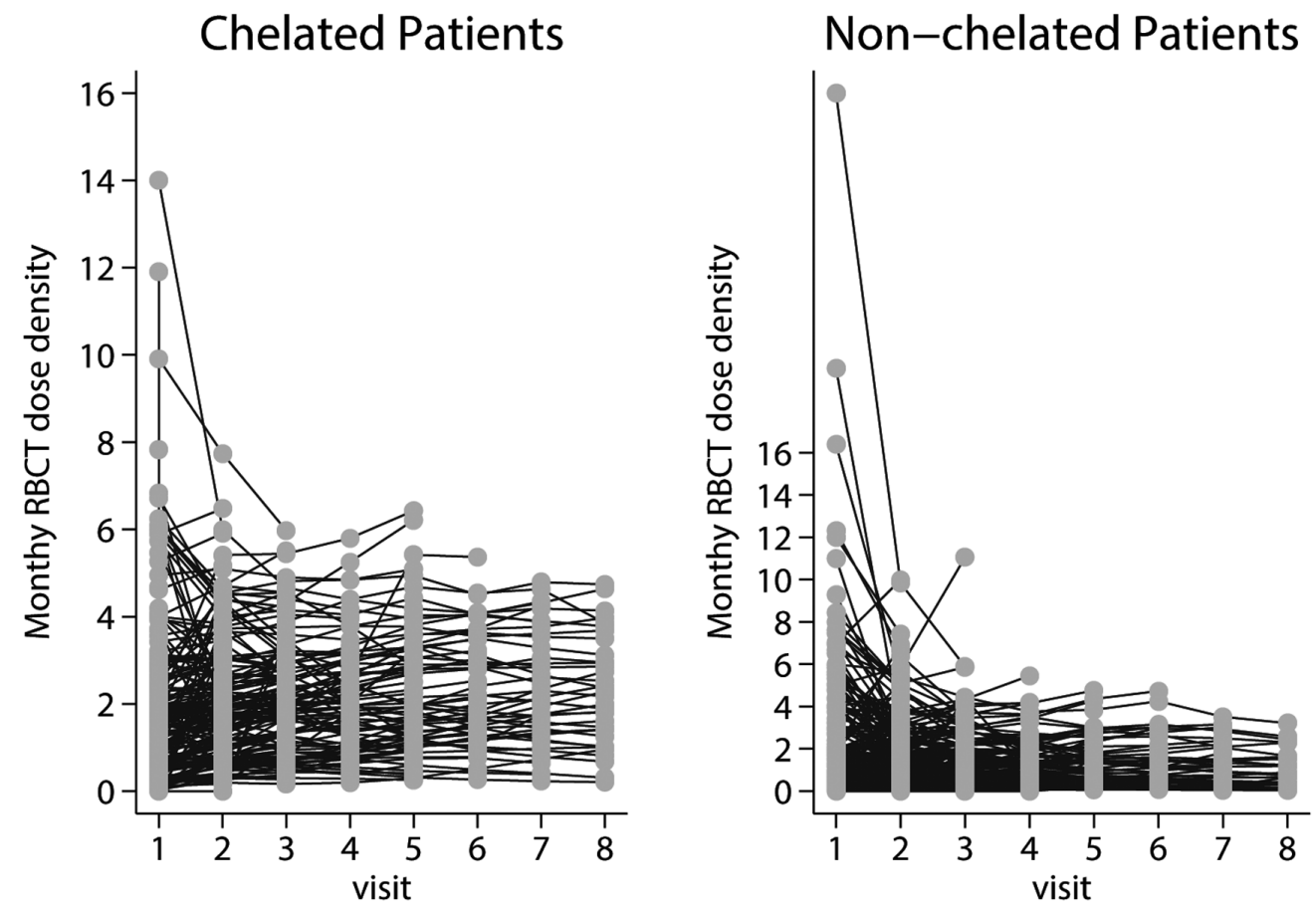

Figure 5. Changes in transfusion density over time in chelated and non-chelated patients. Time: eight 6-monthly visits. 
We observed hematologic responses with all chelating agents.

A subgroup of chelated patients had an at least temporary platelet response $(22.9 \%)$ over time. Median platelet counts were in the normal range in both the chelated and non-chelated group.

Figure $6 \mathrm{~B}$ demonstrates ferritin levels of chelated patients with and without a ferritin response and nonchelated patients. Fifteen $(51.7 \%)$ of the 29 responding patients were treated with ESA and five (17.2\%) were treated with lenalidomide. A subgroup of patients had a ferritin response (5.6-23.5\%) over time. Responding patients showed ongoing mean serum ferritin levels of approximately $1000 \mu \mathrm{g} / \mathrm{L}$, whereas non-responding chelated patients had mean ferritin values of approximately $2100 \mu / \mathrm{L}$.

\section{Chelated patients follow up}

On average, chelated patients did not start therapy until 17 months after diagnosis (Table 1). Of the 199 chelated patients, at the time of the analysis, follow up was ongoing for 148 patients, for seven patients their disease had progressed to higher risk MDS/AML, 29 patients had died, and four have missing values of treatment dates (these four patients are still ongoing), nine patients had withdrawn from the study (four of these because of disease progression and five after starting intensive treatment such as an allogeneic stem cell transplantation), and six were lost to follow up. Most patients (101 of the 148 ongoing patients) were receiving chelation at the time of the last report. Twenty of the 199 chelated patients switched from deferasirox to another chelating agent.
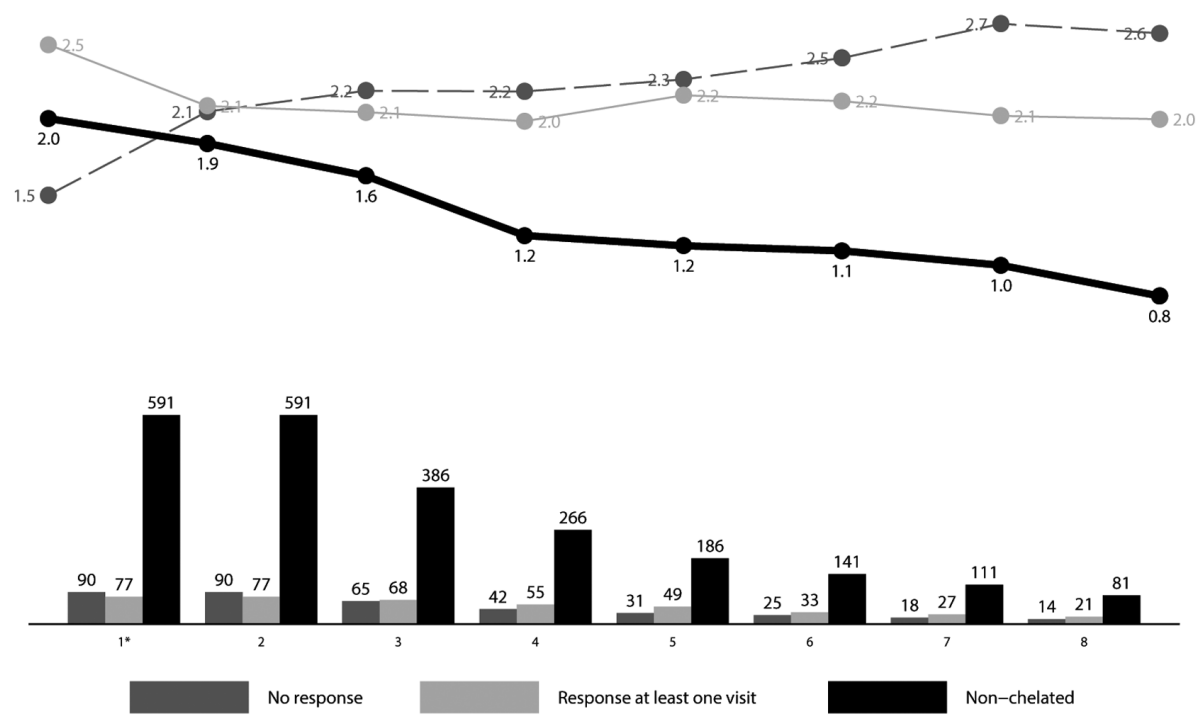

B Ferritin (ug/L)
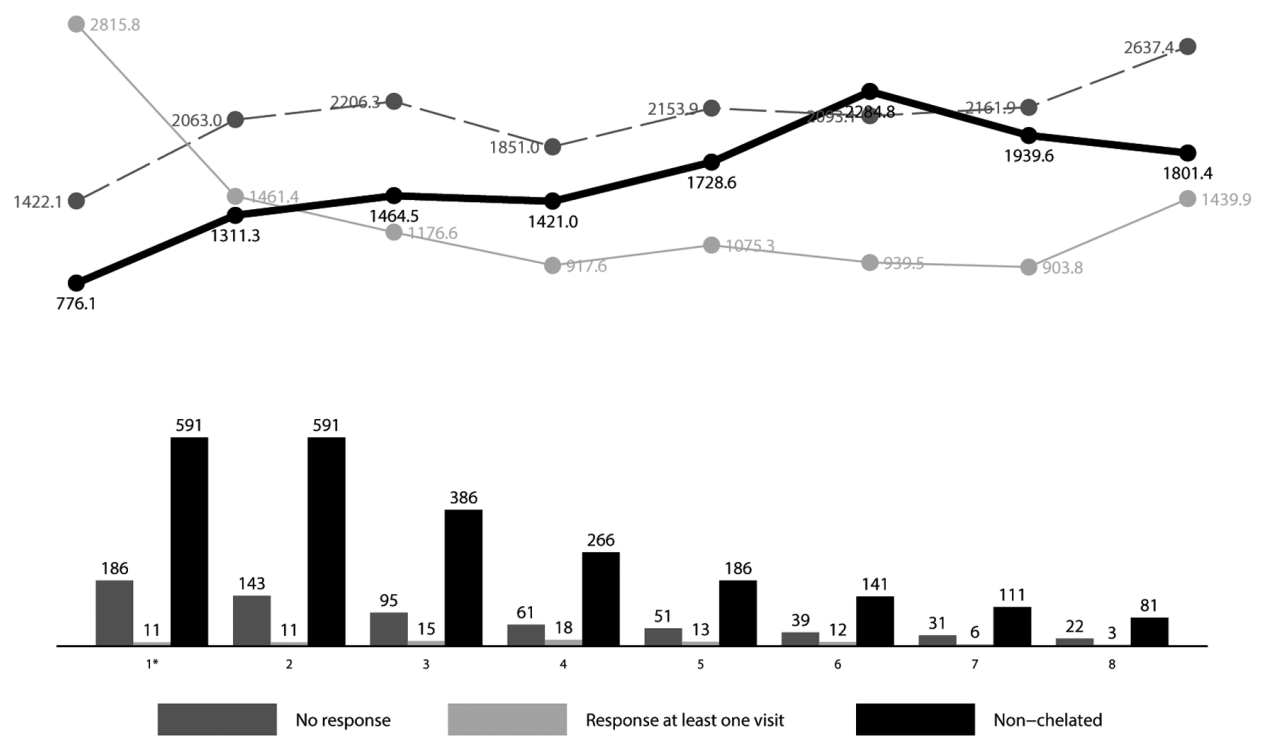

Figure 6. Trajectory analysis in chelated patients with and without response and for nonchelated patients. (A) Monthly red blood cell transfusion density for chelated patients with and without an erythroid response and for non-chelated patients. (B) Ferritin levels of patients with and without a ferritin response, defined as a decrease of $\geq 1000 \mu \mathrm{g} / \mathrm{L}$ or a drop of the serum ferritin value below $1000 \mu \mathrm{g} / \mathrm{L}$, and for nonchelated patients. 

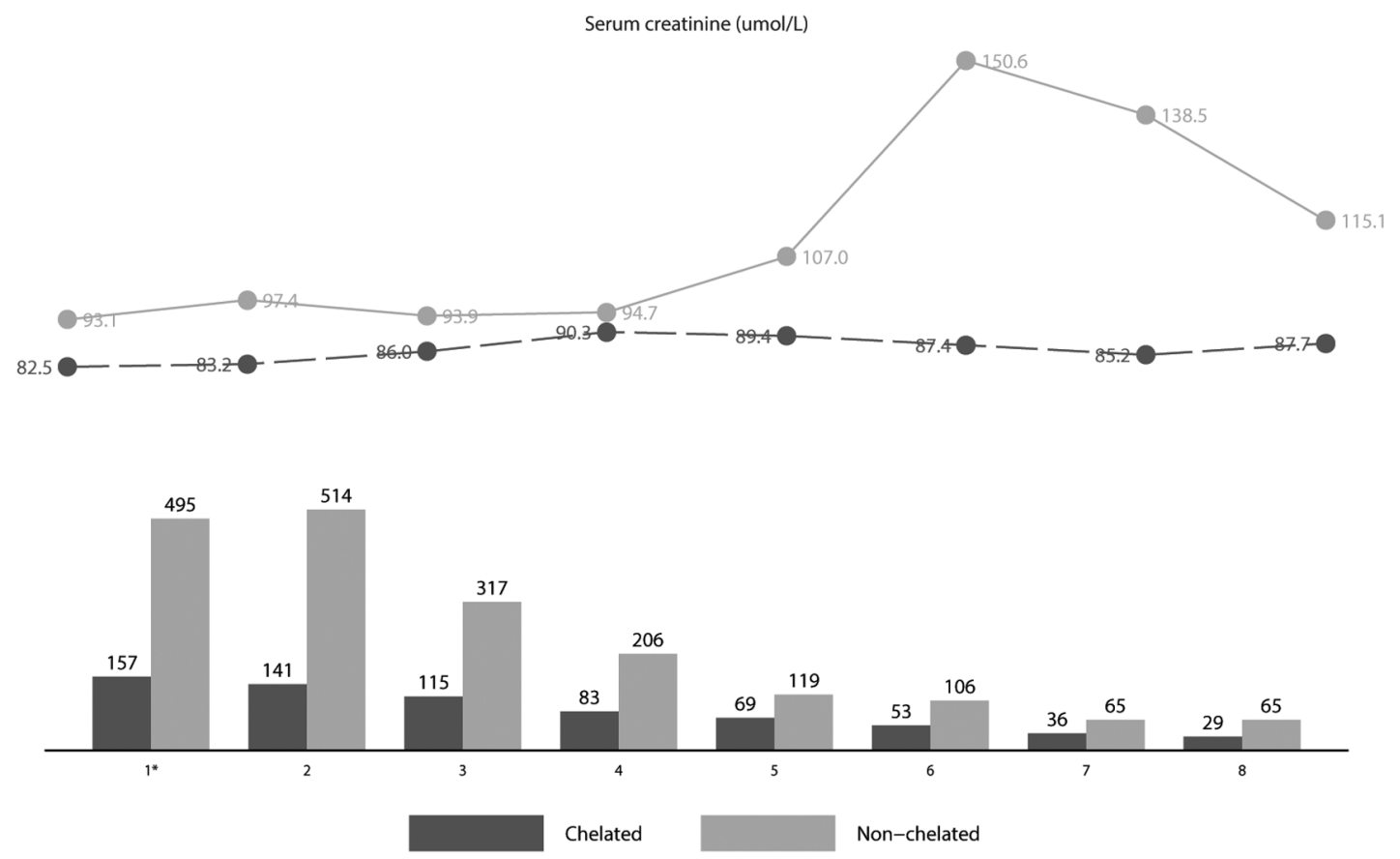

Figure 7. Serum creatinine levels $(\mu \mathrm{mol} / \mathrm{L})$ in chelated and non-chelated patients per check-up.

\section{Reasons for cessation of iron chelation therapy}

Information on reasons of cessation of ICT was not routinely recorded in the study. However, information about the deferasirox-treated patients was available for seven patients: fatigue and diarrhoea (1 patient), physician's choice (1 patient), economic reasons (1 patient), renal failure (1 patient), no effect (1 patient), dyspepsia (1 patient), and lower limb cramps and dosage change (1 patient).

\section{Renal function}

Non-chelated patients had slightly higher median creatinine values compared to chelated patients at time of eligibility [non-chelated: median $86 \mu \mathrm{mol} / \mathrm{L}$ (p10-p90: 61135); chelated: median $79 \mu \mathrm{mol} / \mathrm{L}$ (p10-p90: 59-107)]. Forty-four chelated patients had higher serum creatinine levels at the first visit after discontinuing chelation compared with creatinine levels at time of eligibility $(P=0.02$ for all chelating agents and $P=0.03$ for deferasiox-treated patients). Renal function in non-chelated patients increased similarly over time (Figure 7).

\section{Discussion}

The results of this study indicate that ICT may improve OS in transfusion-dependent lower-risk MDS patients (LR-MDS). Our results are in line with several previously reported studies. ${ }^{7-10,12,25-28}$ Some of these studies attempted to correct for confounding factors, but still suffered from confounding by indication. This generally results in an overestimation of the beneficial effect of ICT on OS in LR-MDS patients. So far, one randomized controlled trial has been reported on this subject. The randomized, placebo-controlled, TELESTO trial ${ }^{29}$ evaluated the event-free survival (EFS) (a composite outcome, including non-fatal events related to cardiac and liver function, and transfor- mation to AML or death) and safety of deferasirox versus placebo in low- and intermediate-1-risk MDS patients. This study demonstrated an EFS risk reduction of $36.4 \%$ in the deferasirox arm $(P=0.015)$. However, there was no difference in median OS in the deferasirox-treated arm (HR 0.83, 95\%CI: 0.54-1.28, $P=0.200$ ) when compared with placebo, but more than $50 \%$ of the placebo-treated patients switched to ICT after study treatment discontinuation (the placebo drug). The results of the TELESTO study are in line with our results. However, the included patients may not represent 'real-life' elderly MDS patients with multiple comorbidities, as reflected by the mean age of 61 years old of the patients included in TELESTO study compared to the mean age of 70 years in the EUMDS Registry study. Furthermore, low accrual rates and the crossover to ICT after cessation of the placebo, affected the statistical power of the TELESTO study.

Meanwhile, well-designed prospective observational data, reflecting, 'real-life' data, contribute to the better understanding of the effect of ICT on OS in LR-MDS patients. Recently, a study from the Canadian MDS registry demonstrated a superior OS for 83 chelated patients compared to non-chelated patients $(5.2$ vs. 2.1 years; $P<0.001){ }^{30}$ The patients in this study were selected at the onset of transfusion dependency. Chelated patients became transfusion-dependent at a much longer interval from diagnosis than non-chelated patients (median $18 \mathrm{vs}$. 6 months) and OS was calculated from the time of becoming transfusion-dependent. Even after matching, some incomparability between the two groups remained in factors like concurrent treatment, presence of ringed sideroblasts, and ferritin levels. Therefore, confounding cannot be excluded in this study. Nevertheless, their conclusions are in accordance with our findings, supporting the probable beneficial effect of ICT on OS in LR-MDS patients. 
The mechanisms by which ICT influences OS after a relatively short exposure to iron chelation therapy (median duration of 13 months) are not completely understood. A recently published study of the EUMDS Registry, as well as the follow-up data of this study, demonstrated detectable labile plasma iron (LPI) levels to be associated with inferior OS in LR-MDS patients. ${ }^{31,32}$ The risk of dying prematurely in patients with detectable LPI levels occurred too early in this study to explain this risk by classical IOL due to organ toxicity (e.g. liver and heart) after long-term transfusions, but this indicates a direct toxic effect associated with elevated LPI levels. ${ }^{31}$

Likewise, there is increasing evidence that increased LPI levels may be a general predictor of an increased nonrelapse mortality during and after hematopoietic stem cell transplantion..$^{33}$

Toxic iron species are known to catalyze the cellular generation of ROS, which play a key role in cellular damage. ${ }^{34,35}$ ROS damage (mitochondrial) DNA, with potential consequent genomic instability, mutagenesis, and cell death. ROS are associated with leukemic transformation of the MDS clone. ${ }^{6}$ Moreover, ICT is associated with a decrease in LPI and ROS. ${ }^{6,16}$ Overall, the present study indicates that ICT may partly counteract the unfavorable consequences of secondary IOL.

In up to $31.0 \%$ of chelated patients a reduction in transfusion density was observed during at least one interval between check-ups. Likewise, $27.4 \%$ of the responding patients became, at least temporarily, transfusion independent. Platelet responses were less frequently observed. However, platelet count in this context was less relevant because the platelet counts in both groups were within the normal range, and will not likely lead to severe bleeding complications. Contemporary treatment with ESA and/or lenalidomide may have enhanced these responses.

Several previous studies recorded hematologic responses to ICT. ${ }^{11-16}$ While the percentage of patients with hematologic responses in these studies are in line with the present study, none of the former studies included a control group in their analyses. One of the key factors is the relatively short period of ICT (median 13 months) in this study. The duration of ICT may improve by the introduction of a better tolerated formulation of deferasirox. ${ }^{36}$ ICT is usually prescribed relatively late after detection of signs of IOL. Earlier initiation of ICT may prevent or decrease the occurrence of transfusional iron toxicity on hematopoiesis. Moreover, we recorded data only at 6monthly intervals. Short duration hematologic responses in between check-ups may be missed by this approach. But on the other hand, short-term responses may not be clinically relevant.

Pre-clinical studies have shown a beneficial effect of ICT on hematopoiesis. ${ }^{35,37}$ Inhibition of the transcription factor NF-kB, involved in many cellular processes, and modulation of mammalian target of rapamycin (M-TOR) signaling, a major regulator of cell death and proliferation, have been proposed to play a role. ${ }^{17}$ Future studies should address this issue appropriately.

In the trajectory analyses, ferritin responses occurred in up to $23.5 \%$ of the chelated patients. Serum ferritin levels have frequently been reported to be a prognostic marker in LR-MDS patients, but serum ferritin is an imprecise surrogate marker for secondary IOL and toxicity. ${ }^{38,39}$ This is reflected by the observation that a relatively small pro- portion of chelated patients have a considerable decrease in serum ferritin levels, while these patients show a significant survival benefit. Serum ferritin levels are influenced by the stage of MDS and by concurrent infection and inflammation, which is common in LR-MDS patients..$^{38}$ In addition, there is no convincing evidence regarding its use for monitoring secondary IOL in MDS patients. ${ }^{8,40}$ Tissue biopsy and MRI T2* are currently regarded to be the most specific and sensitive diagnostic tests for detecting IOL. ${ }^{38}$ However, the clinical utility of these assays remains unclear in MDS and invasiveness (biopsy), unavailability, and expense (MRI T2*) hamper their general use in clinical practice. As discussed above, LPI is associated with inferior survival in LR-MDS patients. ${ }^{11,32}$ Future studies are warranted to evaluate the effect of ICT on LPI levels as a measure of iron toxicity. Measurement of oxidative stress, including malondialdehyde, a long-lasting lipid peroxidation product, formed as a consequence of oxidative stress from IOL, are also possible future markers to detect and monitor the biological consequences of secondary IO in LR-MDS patients, should they be proven to correlate with clinical outcomes. ${ }^{41,42}$

Analysis of renal function demonstrated that ICT is associated with an increase in creatinine levels. In some patients, this will be clinically relevant and/or a reason to stop or lower the dose of ICT. In other patients, an increase in creatinine levels will not affect cessation of ICT.

This large cohort, with prospectively collected 'real-life' data from diagnosis, provides a unique opportunity to study the effect of ICT in a large number of lower-risk MDS patients in daily practice. An important strength of the study is that the results can be widely generalized to this, mostly elderly, patient population with multiple comorbidities, who are typically excluded from clinical trials. The variation in iron chelation practice across the different countries, due to variable interpretation of the poor quality outcome data for ICT in MDS, made it possible to compare the effects of ICT on OS to a non-chelated control group. In Europe, unlike in the United States, socio-economic status does not influence the prescription of ICT (either deferoxamine or deferasirox) because the costs are covered by the national health systems.

Since conventional statistical modeling is limited by the number of co-variates to be added to a model, propensity-score matched analysis is able to incorporate more confounding factors in the model, including country-specific effects. Confounding by indication, a common problem in observational studies, is maximally reduced by using the propensity-score matched method and is, therefore, a major strength of this study. To our knowledge, we are the first to apply this method in order to adequately deal with confounding in this setting.

Limitations of our study include the moderate sample size of the deferoxamine and deferiprone groups. In addition, our analysis could not consider differences in dosing schemes and therapy compliance. This prevented us from drawing definitive conclusions of the effect of the separate iron chelators on OS. Data were collected at the scheduled 6-monthly intervals. Subtle changes in patientrelated factors in the intervening 6-month period may have been missed. Not all patients can be matched by the propensity score approach. This might introduce selection bias. However, the same magnitude and direction of 
the results were seen in the analysis of the unmatched samples. Therefore, in this case, propensity-score matching will probably not have led to significant selection bias. Finally, despite using a large control group, eligible for using ICT, and a propensity-score matched analysis corrected for many known and measured confounders, we cannot exclude residual confounding. Considering the size of the effect, it is unlikely that residual confounding would explain the difference found between chelated and non-chelated patients.

In summary, the results of this study suggest that ICT may improve OS and hematopoiesis in transfused LRMDS patients.

\section{Acknowledgments}

The authors and members of the steering committee of the EUMDS registry would like to thank all local investigators and operational team members for their contribution to the registry, and W. Thomas Johnston for his contribution to the analyses.

\section{Funding}

The EUMDS Registry is supported by an educational grant from Novartis Pharmacy B.V. Oncology Europe, and Amgen Limited. This work is part of the MDS-RIGHT activities, which has received funding from the European Union's Horizon 2020 research and innovation programme under grant agreement No 634789 - "Providing the right care to the right patient with MyeloDysplastic Syndrome at the right time".

\section{References}

1. Nimer SD. Myelodysplastic syndromes. Blood. 2008;111(10):4841-4851.

2. Porter J, Garbowski M. Consequences and management of iron overload in sickle cell disease. Hematology Am Soc Hematol Educ Program. 2013;2013:447-456.

3. Olivieri NF, Brittenham GM. Iron-chelating therapy and the treatment of thalassemia. Blood. 1997;89(3):739-761.

4. Powell LW, Seckington RC, Deugnier Y. Haemochromatosis. Lancet. 2016; 388(10045):706-716.

5. Greenberg P, Cox C, LeBeau MM, et al. International scoring system for evaluating prognosis in myelodysplastic syndromes. Blood. 1997;89(6):2079-2088.

6. Porter JB, de Witte T, Cappellini MD, et al. New insights into transfusion-related iron toxicity: Implications for the oncologist. Crit Rev Oncol Hematol. 2016;99:261-271.

7. Rose C, Brechignac S, Vassilief D, et al. Does iron chelation therapy improve survival in regularly transfused lower risk MDS patients? A multicenter study by the GFM (Groupe Francophone des Myélodysplasies). Leuk Res. 2010; 34(7):864-870.

8. Raptis A, Duh MS, Wang ST, et al. Treatment of transfusional iron overload in patients with myelodysplastic syndrome or severe anemia: data from multicenter clinical practices. Transfusion. 2010;50(1):190199.

9. Neukirchen J, Fox F, Kündgen A, et al. Improved survival in MDS patients receiving iron chelation therapy - a matched pair analysis of 188 patients from the Düsseldorf MDS registry. Leuk Res. 2012; 36(8):1067-1070

10. Delforge M, Selleslag D, Beguin Y, et al. Adequate iron chelation therapy for at least six months improves survival in transfusion-dependent patients with lower risk myelodysplastic syndromes. Leuk Res. 2014;38(5):557-563.

11. Messa E, Biale L, Castiglione A, et al. Erythroid response during iron chelation therapy in a cohort of patients affected by hematologic malignancies and aplastic anemia with transfusion requirement and iron overload: a FISM Italian multicenter retrospective study. Leuk Lymphoma. 2017;58(11):2752-2754.

12. Angelucci E, Santini V, Di Tucci AA, et al. Deferasirox for transfusion-dependent patients with myelodysplastic syndromes: safety, efficacy, and beyond (GIMEMA MDS0306 Trial). Eur J Haematol. 2014;92(6):527-536.

13. Nolte F, Höchsmann B, Giagounidis A, et al. Results from a 1-year, open-label, single arm, multi-center trial evaluating the efficacy and safety of oral Deferasirox in patients diagnosed with low and int-1 risk myelodysplastic syndrome (MDS) and transfusion-dependent iron overload. Ann Hematol. 2013;92(2):191-198.

14. Jensen PD, Heickendorff L, Pedersen B, et al. The effect of iron chelation on haemopoiesis in MDS patients with transfusional iron overload. Br J Haematol. 1996;94(2):288-299.

15. List AF, Baer MR, Steensma DP, et al. Deferasirox reduces serum ferritin and labile plasma iron in RBC transfusiondependent patients with myelodysplastic syndrome. J Clin Oncol. 2012;30(17):21342139.

16. Gattermann N, Finelli C, Della Porta M, et al. Hematologic responses to deferasirox therapy in transfusion-dependent patients with myelodysplastic syndromes. Haematologica. 2012;9(9):1364-1371.

17. Breccia M, Voso MT, Aloe Spiriti MA, et al. An increase in hemoglobin, platelets and white blood cells levels by iron chelation as single treatment in multitransfused patients with myelodysplastic syndromes: clinical evidences and possible biological mechanisms. Ann Hematol. 2015;94(5):771-777.

18. Bennett JM. World Health Organization classification of the acute leukemias and myelodysplastic syndrome. Int J Hematol. 2000:72(2):131-133.

19. Caliendo M, Kopeinig S. SOME PRACTICAL GUIDANCE FOR THE IMPLEMENTATION OF PROPENSITY SCORE MATCHING. Journal of Economic Surveys. 2008;22(1):31-72.

20. Rosenbaum PR, Rubin DB. Constructing a Control Group Using Multivariate Matched Sampling Methods That Incorporate the Propensity Score. The American Statistician. 1985;39(1):33-38.

21. Stürmer T, Joshi M, Glynn RJ, et al. A review of the application of propensity score methods yielded increasing use, advantages in specific settings, but not substantially different estimates compared with conventional multivariable methods. J Clin Epidemiol. 2006;59(5):437-447.

22. Schafer JL. Analysis of Incomplete
Multivariate Data: Taylor \& Francis, 1997. 23. Schultz LR, Peterson EL, Breslau N Graphing survival curve estimates for timedependent covariates. Int $\mathrm{J}$ Methods Psychiatr Res. 2002;11(2):68-74.

24. Cheson BD, Greenberg PL, Bennett JM, et al. Clinical application and proposal for modification of the International Working Group (IWG) response criteria in myelodysplasia. Blood. 2006;108(2):419425.

25. Zeidan AM, Hendrick F, Friedmann E, et al Deferasirox therapy is associated with reduced mortality risk in a medicare population with myelodysplastic syndromes. J Comp Eff Res. 2015;4(4):327-340

26. Mainous AG, Tanner RJ, Hulihan MM, et al. The impact of chelation therapy on survival in transfusional iron overload: a metaanalysis of myelodysplastic syndrome. $\mathrm{Br} \mathrm{J}$ Haematol. 2014;167(5):720-723.

27. Remacha Á, Arrizabalaga B, Villegas A, et al. Evolution of iron overload in patients with low-risk myelodysplastic syndrome: iron chelation therapy and organ complications. Ann Hematol. 2015;94(5):779-787.

28. Lyons RM, Marek BJ, Paley C, et al. Relation between chelation and clinical outcomes in lower-risk patients with myelodysplastic syndromes: Registry analysis at 5 years. Leuk Res. 2017;56:8895.

29. Angelucci E, Li J, Greenberg PL, et al. Safety and efficacy, including event-free survival, of deferasirox versus placebo in iron-overloaded patients with low- and intermediate-1-risk myelodysplastic syndromes (MDS): outcomes from the randomized double blind Telesto study. Blood, annual meeting abstracts. 2018;132(S1):abstract 234.

30. Leitch HA, Parmar A, Wells RA, et al Overall survival in lower IPSS risk MDS by receipt of iron chelation therapy, adjusting for patient-related factors and measuring from time of first red blood cell transfusion dependence: an MDS-CAN analysis. Br J Haematol. 2017;179(1):83-97.

31. de Swart L, Reiniers C, Bagguley T, et al Labile plasma iron levels predict survival in patients with lower-risk Myelodysplastic syndromes. Haematologica. 2018; 103(1):69-79.

32. Hoeks M, Bagguley T, Roelofs R, et al. Elevated labile plasma iron (LPI) levels in patients with lower-risk myelodysplastic syndromes (MDS) are associated with 
decreased quality of life and reduced overall survival. Blood, annual meeting abstracts. 2018;132(S1):abstract 4392.

33. Wermke M, Eckholdt J, Götze KS, et al. Enhanced labile plasma iron and outcome in acute myeloid leukaemia and myelodysplastic syndrome after allogeneic haemopoietic cell transplantation (ALLIVE): a prospective, multicentre, observational trial. Lancet Haematol. 2018; 5:e201-210.

34. Hershko C, Link G, Cabantchik I. Pathophysiology of iron overload. Ann N Y Acad Sci. 1998;850:191-201.

35. Pilo F, Angelucci E. A storm in the niche: Iron, oxidative stress and haemopoiesis. Blood Rev. 2018;32(1):29-35.

36. Taher AT, Saliba AN, Kuo KH, et al. Safety and Pharmacokinetics of the Oral Iron Chelator SP-420 in $\beta$-thalassemia. Am J Hematol. 2017;92(12):1356-1361.

37. Hartmann J, Braulke F, Sinziq U, et al. Iron overload impairs proliferation of erythroid progenitors cells (BFU-E) from patients with myelodysplastic syndromes. Leuk Res. 2013;37(3):327-332.

38. Cazzola M, Della Porta MG, Malcovati L. Clinical relevance of anemia and transfusion iron overload in myelodysplastic syndromes. Hematology Am Soc Hematol Educ Program. 2008:166-175.

39. Malcovati L, Porta MG, Pascutto C, et al. Prognostic factors and life expectancy in myelodysplastic syndromes classified according to WHO criteria: a basis for clinical decision making. J Clin Oncol. 2005,
23(30):7594-7603.

40. Greenberg PL, Attar E, Bennett JM, et al NCCN Clinical Practice Guidelines in Oncology: myelodysplastic syndromes. J Natl Compr Canc Netw. 2011;9(1):30-56.

41. Pimková K, ChrastinováL, Suttnar J, et al. Plasma levels of aminothiols, nitrite, nitrate, and malondialdehyde in myelodysplastic syndromes in the context of clinical outcomes and as a consequence of iron overload. Oxid Med Cell Longev. 2014 Jan; DOI 10.1155/2014/416028.

42. de Souza GF, Barbosa MC, Santos TE, et al. Increased parameters of oxidative stress and its relation to transfusion iron overload in patients with myelodysplastic syndromes. J Clin Pathol. 2013;66(11):996998. 